\title{
Ignition First in a Fusion Reaction
}

\author{
In August, a fusion reaction at the National Ignition Facility yielded a \\ record $1.3 \mathrm{MJ}$ in fusion energy, releasing, for the first time, more energy \\ than the fuel capsule absorbed.
}

\section{By Katherine Wright}

$f$ they realize their full potential, nuclear fusion reactors could provide the world with a near limitless amount of clean electricity. That potential is still far from being achieved. But speaking at the recent Annual Meeting of the APS Division of Plasma Physics, Debbie Callahan of Lawrence

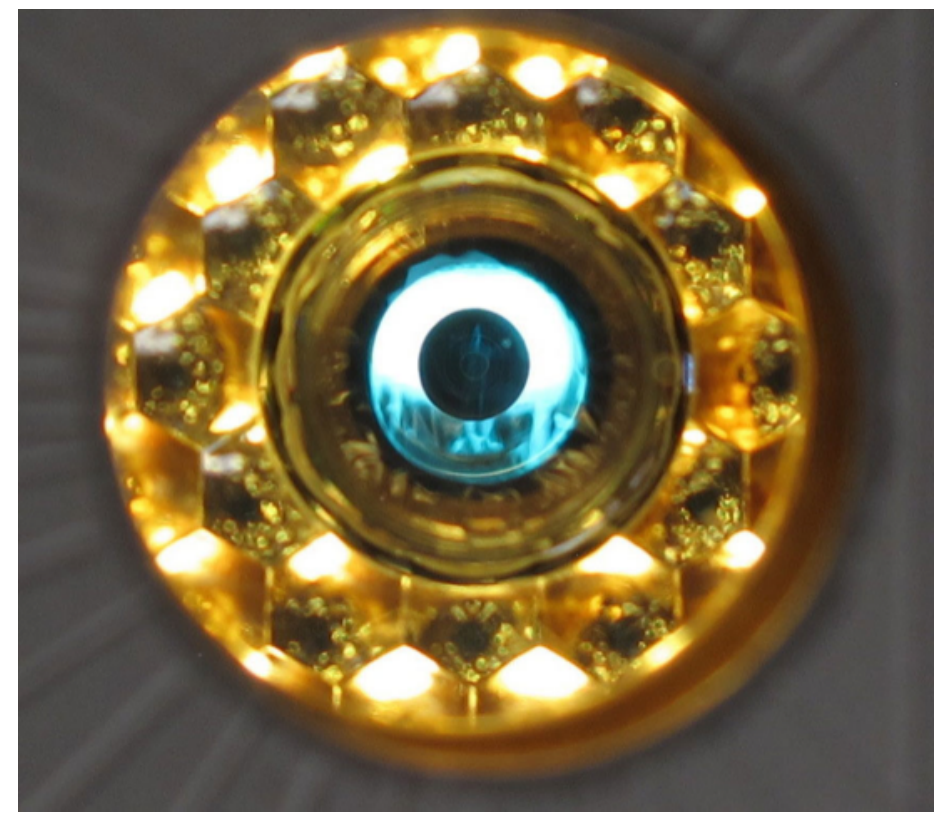

A photo of one of the targets used at the National Ignition Facility. The spherical capsule where thermonuclear fuel is loaded can be seen in the middle. The outer cylinder is the hohlraum, which converts incoming laser light into $x$ rays that bombard the capsule with heat.

Credit: Lawrence Livermore National Laboratory
Livermore National Laboratory (LLNL), California, announced that researchers have, for the first time, triggered "ignition"-a fusion reaction that produces more energy than it receives and can thus burn on its own. The demonstration, which was performed at LLNL's National Ignition Facility (NIF), takes commercial fusion-energy reactors a step closer to reality. It also provides a platform for understanding materials under extreme conditions.

So-called inertial confinement fusion, the type of fusion approach being studied at NIF, involves rapidly imploding a millimeter-sized capsule filled with a thermonuclear fuel mixture of deuterium and tritium (two forms of hydrogen). The capsule is heated with $x$ rays generated by high-power lasers, turning the capsule into a plasma. This plasma accelerates inward, like a collapsing star, compressing the capsule's deuterium-tritium fuel into a tiny sphere with a temperature exceeding 100 million degrees Celsius and a pressure more than 100 billion times greater than that of Earth's atmosphere. Under such conditions, hydrogen atoms in the fuel fuse, releasing energy.

For a commercial fusion reactor, these fusion reactions need to be self-sustaining, meaning that they need to heat the plasma enough to induce additional fusion reactions. This self-sustaining condition is fundamentally what is meant by ignition, says plasma physicist Jeremy Chittenden from Imperial College London. But "it's really difficult to diagnose directly what's happening inside the fuel," he says. So scientists have adopted more practical definitions of ignition based on the outgoing energy from fusion being greater than the 


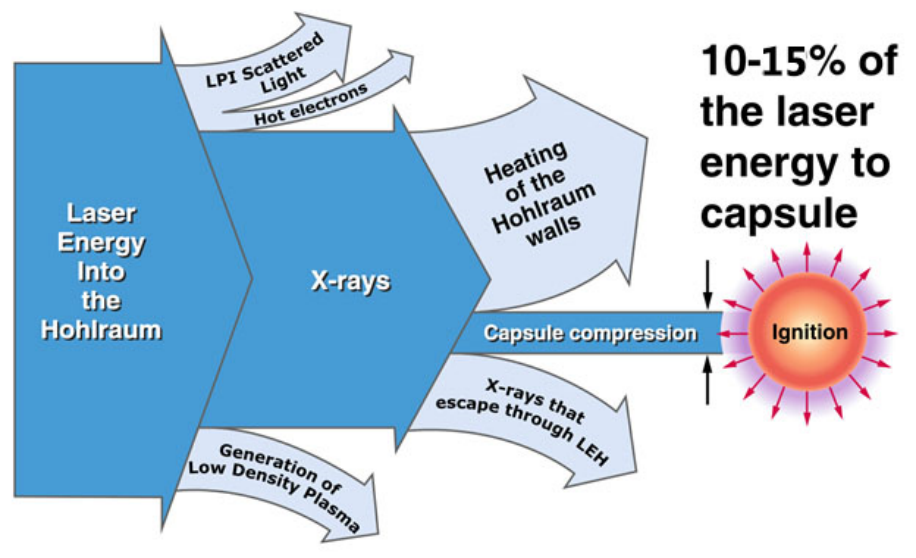

Simplified flow diagram showing where the "laser energy" goes in a NIF experiment. Only about $150-270 \mathrm{~kJ}$ reaches the fuel capsule, with most of the losses going into heating the walls of the hohlraum.

Credit: Lawrence Livermore National Laboratory

incoming energy from external heating sources.

Until August of this year, no facility had achieved this ignition threshold. That changed on August 8th, with an upgraded experiment that yielded $1.3 \mathrm{MJ}$, which is about 8 times more energy than NIF's previous record. Early reports referred to this breakthrough as the "brink of ignition," because it yielded less than the $1.9 \mathrm{MJ}$ supplied by the facility's 192 laser beams. But many fusion scientists think such a perspective is too conservative. "As far as most people working in the field are concerned, the scientific demonstration of the ignition process has indeed been achieved," Chittenden says.

The argument for ignition is based on an accounting of losses in the energy delivery: Calculations show that only about $230 \mathrm{~kJ}$ of the laser energy reaches the fuel capsule. For Callahan, this last energy is the relevant one to consider, as it describes the heat coming in from outside. "We got out almost 6 times as much energy as we put into the capsule," she says. "It's a big accomplishment." She adds that achieving ignition is "what I set out to do" in becoming a fusion scientist, "and we did it."

The question now is, what brought about this big boost in output? The latest experiment trialed several advances in the equipment, fuel, and methods. These advances included creating a fuel-capsule shell with fewer defects and using a significantly narrower tube to place the deuterium-tritium fuel inside the capsule. Callahan says that both changes likely reduced the size of the instabilities that formed within the shell as it turned into a plasma. If those instabilities are too large, they can lead to higher-atomic-number material being injected into the fuel, disrupting fusion.

The NIF scientists also made some other design tweaks that allowed them to increase the speed at which the capsule shell imploded. A faster accelerating shell transfers more energy to the fuel hotspot when the implosion is halted by the internal pressure, Callahan says. She compares it to stopping a car with brakes: The faster the car is moving, the hotter the brakes get. "We wanted to slam the brakes on as hard as possible to transfer as much energy as possible from the shell to the [fuel] hotspot," she says.

So are commercial fusion reactors now just around the corner? Not according to Callahan, who calls this advance a "key step down a long road." To make a viable commercial fusion reactor, the reaction needs to produce significantly more energy than the reactor requires to run. To create the 1.9-MJ-laser input at $\mathrm{NIF}$ requires around $400 \mathrm{MJ}$ of electricity. And much of that laser energy is lost before it reaches the hydrogen fuel. One place for significant loss is in a metal cylinder, called a hohlraum, that surrounds the capsule. The hohlraum converts the laser light into $x$ rays that transport the laser energy to the fuel. However, a large fraction of this input energy is lost to heating the hohlraum walls.

Reducing the fraction of energy lost in the hohlraum is on the near-term to-do list for NIF. Nuclear fusion scientists are also exploring new avenues for research opened up by the reaching of a new experimental regime. "It's an extremely exciting time to be working on this topic," Callahan says. "Our field is really in a place that we've never been before."

Katherine Wright is the Deputy Editor of Physics. 